survival for bDMARD naïve vs. non-naïve patients was similar for all diagnoses (RA, $p=0.15 ; \mathrm{PsA}, \mathrm{p}=0.23$; ax-SpA $p=0.71$ ), with trend towards better drug survival in bDMARD naïve RA and PsA patients (figure). 4 year drug survival in bDMARD naïve/non-naïve patients were: RA, 54/48\%; PsA, 47/43\%; ax-SpA, 48/46\%, respectively. Subgroup analyses of patients with and without concomitant sDMARDs showed similar findings. A trend was seen towards better 3 month responses in bDMARD naïve vs. non-naïve patients, with statistically significant better responses for DAS28 in PsA and BASDAI and ASDAS in ax-SpA (table 1).

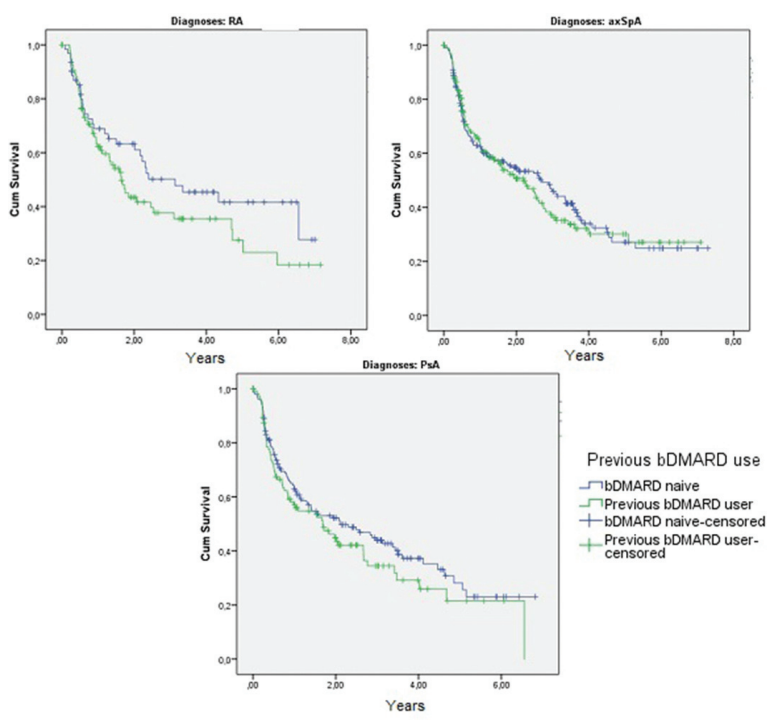

Conclusions: Golimumab drug survival was similar in bDMARD naïve vs. nonnaïve RA, PsA and ax-SpA patients. A trend was seen towards better responses for bDMARD naïve patients. Identified predictors for golimumab drug discontinuation was female gender and no concomitant sDMARDs in PsA and female gender in ax-SpA.

Disclosure of Interest: B. Michelsen Consultant for: Novartis, J. Sexton: None declared, T. Kvien Grant/research support from: AbbVie, BMS, MSD, Pfizer, Roche and UCB, Consultant for: AbbVie, Biogen, BMS, Boehringer Ingelheim, Celgene, Celltrion, Eli Lilly, Epirus, Hospira, Merck-Serono, MSD, Mundipharma, Novartis, Oktal, Orion Pharma, Hospira/Pfizer, Roche, Sandoz and UCB DOI: 10.1136/annrheumdis-2018-eular.1885

\section{SAT0265 THE RESPONSE TO TNF-BLOCKERS TREATMENT OF SPA PATIENTS IS INFLUENCED BY THE INTERPLAY BETWEEN HLA-B27 AND GUT MICROBIOTA COMPOSITION AT BASELINE}

M. Vallier ${ }^{1}$, M. Chamaillard ${ }^{1}$, S. Ferreira ${ }^{2}$, S. Menegatti ${ }^{3}$, E. Bianchi $^{3}$, L. Rogge ${ }^{3}$, M. Dougados ${ }^{4,5}$, C. Miceli-Richard ${ }^{3,4} .{ }^{1} N L R I I$, Institut Pasteur, ${ }^{2}$ Genoscreen, Lille; ${ }^{3}$ Immunoregulation Unit 25, Institut Pasteur, ${ }^{4}$ Service de Rhumatologie, Hôpital Cochin; ${ }^{5}$ Inserm U1153, Clinical Epidemiology and Biostatistics, PRES Sorbonne Paris-Cité, Paris, France

Background: The response to TNF-blockers in axial spondyloarthritis ( $\mathrm{AxSpA}$ ) is at least partially influenced by HLA-B27 through a still poorly understood mechanism.

Objectives: Given that HLA-B27 regulates the gut microbiota composition in rats $^{1,2}$, we seek to evaluate the predictive value of the gut microbiota composition in AxSpA patients on their responsiveness to TNF-blockers.

Methods: A total of 58 patients was monocentrically recruited between October 2014 and May 2015. At baseline, these patients had an active disease despite NSAIDs intake and were eligible for treatment with a TNF-blocker, while having no history of inflammatory bowel disease (IBD). The mean BASDAI ( \pm SD) was 45.6 \pm 21.4 ; ASDAS $2.8 \pm 0.9$ and CRP $9.7 \pm 11.4 \mathrm{mg} / \mathrm{L}$. Among these patients, 56 fulfilled the ASAS classification criteria (imaging arm) with sacro-ilitis on X-rays $(n=37)$ or objective signs of inflammation on MRI $(n=48)$. Two patients fulfilled the clinical arm. These patients were not subjected to antibiotics within 3 months before stool sample collection. Bacterial 16S rRNA gene sequencing of the V3-V4 region was performed on stools samples before and 3 months after TNF-blocker treatment. Beta diversity metrics were calculated on the abundance of operational taxonomic units (OTU) after their taxonomic assignment on quality-filtered sequences.

Results: Principal component analysis (PCA) ordination of Bray-Curtis similarity revealed that current smoking (compared with never or ever smokers) and HLAB27 genotype were significantly associated with the overall composition of the microbiota at baseline. Meanwhile, the abundance of eleven bacterial OTUs was influenced by HLA-B27 genotype at baseline but not after 3 month of treatment. In contrast, we identified a bacterial signature that was linked to the smoking behaviour independently of TNF-blocker treatment, whereas the BASDAI and ASDAS indices were significantly associated to the general composition of the gut microbiota after the 3 month treatment. In line with a previous report ${ }^{3}$, the abundance of Ruminococcus gnavus was not associated with disease activity in the absence of IBD. Interestingly, the abundance of 5 and 7 bacterial OTUs at baseline was associated with the response to TNF-blockers assessed by BASDAI and ASDAS, respectively. Among these candidates, the abundance of one bacterial OTUs belonging to the Clostridiales order was associated with a better response to the treatment and with the HLA-B27 genotype.

Conclusions: Anti-TNF treatment was found to modulate the HLA-B27-induced variations of the intestinal microbiota of AxSpA patients. Moreover, the abundance of a subset of OTUs at baseline was found to predict the responsiveness to TNF-blockers. Further functional studies will be conducted to assess how these taxa can be use as predictors of the treatment outcome.

\section{REFERENCES :}

[1] Gill, et al. Effects of HLA-B27 on Gut Microbiota in Experimental Spondyloarthritis Implicate an Ecological Model of Dysbiosis. Arthritis Rheumatol 2017

[2] Lin, et al. HLA-B27 and human $\beta 2$-microglobulin affect the gut microbiota of transgenic rats. PLoS One 2014.

[3] Breban, et al. Faecal microbiota study reveals specific dysbiosis in spondyloarthritis. Annal Rheum Dis 2017.

Disclosure of Interest: None declared

DOI: 10.1136/annrheumdis-2018-eular.5208

\section{SAT0266 USE OF CONVENTIONAL SYNTHETIC DMARDS AND BIOLOGICAL DMARDS IN PATIENTS WITH ENTEROPATHIC SPONDYLOARTHRITIS: A COMBINED GASTRO-RHEUMATOLOGICAL APPROACH}

M.S. Chimenti ${ }^{1}$, P. Conigliaro ${ }^{1}$, P. Triggianese ${ }^{1}$, C. Canofari ${ }^{1}$, F. Cedola ${ }^{1}$, S. Onali ${ }^{1}$ E. Calabrese ${ }^{1}$, C. Petruzziello ${ }^{1}$, A. Ruffa ${ }^{1}$, G. Monteleone ${ }^{2}$, L. Biancone ${ }^{2}$,

R. Perricone ${ }^{1}$. ${ }^{1}$ Department of system medicine, Rheumatology, Allergology and Clinical Immunology; ${ }^{2}$ Department of systems medicine, Unit of Gastroenterology, University of Rome Tor Vergata, Roma, Italy

Background: Enteropathic spondyloarthritis (eSpA) is a chronic autoimmune disease associated with inflammatory bowel disease (IBD) that is poorly diagnosed and managed.

Objectives: To assess the diagnostic and therapeutic effect of a combined gastro-rheumatological approach in $\mathrm{SPA}$ patients.

Methods: IBD-patients with joint pain referred to a dedicated rheumatologist by gastroenterologist were enrolled. Clinical and biochemical variables, SpA and intestinal disease activity measures, and treatment (biologic; bDMARDs and conventional synthetic; csDMARDs) were recorded at baseline, 3, 6, 12 and 24 months. The association between treatment on demographic and clinical characteristics was evaluated by logistic regression.

Results: From a total of 229 IBD patients, 147 (64.2\%) were diagnosed with eSpA; $96(65.3 \%)$ showing peripheral involvement and 51 (34.7\%) with axial involvement. The majority $(67.3 \%)$ of eSpA patients were female $(n=99)$, median age and disease duration of 46 and 14.6 years. bDMARD treatment increased over the follow-up period (baseline-24 months: $32.6 \%>60 \%$; AOR:3.45, $95 \% \mathrm{Cl}$ : 1.93-6.2, $p<0.001)$, however, their use was less frequent in elderly patients (AOR: $0.73,95 \% \mathrm{Cl}: 0.56-0.96, \mathrm{p}=0.023$ ), in ulcerative colitis patients (AOR:0.43, $95 \% \mathrm{Cl}: 0.2-0.94, \quad \mathrm{p}=0.034)$ and in patients with peripheral involvement (AOR:0.53, 95\% Cl:0.3-1.04, $\mathrm{p}=0.067$ ). csDMARD use was increased in patients with peripheral involvement (AOR: 4.65, 95\% Cl:2.09-10.33, $\mathrm{p}<0.001$ ) and in patient with ulcerative colitis (AOR:2.30, 95\% Cl:1.13-4.67, $\mathrm{p}=0.021$ ) (figure 1). CRP, ESR, ASDAS-ESR levels and BASFI were significantly decreased over the follow-up period whereas pMayo score, BASDAI and HAQ-S were unchanged (figure 2). 